\title{
Optimal capital injections and dividends with tax in a risk model in discrete time
}

\section{Katharina Bata ${ }^{1} \cdot$ Hanspeter Schmidli ${ }^{2}$}

Received: 29 March 2019 / Revised: 29 October 2019 / Accepted: 18 December 2019 /

Published online: 6 January 2020

(c) The Author(s) 2020

\begin{abstract}
We consider a risk model in discrete time with dividends and capital injections. The goal is to maximise the value of a dividend strategy. We show that the optimal strategy is of barrier type. That is, all capital above a certain threshold is paid as dividend. A second problem adds tax to the dividends but an injection leads to an exemption from tax. We show that the value function fulfils a Bellman equation. As a special case, we consider the case of premia of size one. In this case we show that the optimal strategy is a two barrier strategy. That is, there is a barrier if a next dividend of size one can be paid without tax and a barrier if the next dividend of size one will be taxed. In both models, we illustrate the findings by de Finetti's example.
\end{abstract}

Keywords Discrete risk model - Optimal dividend problem - Capital injections · Tax $\cdot$ Bellman equation $\cdot$ Two barrier strategy $\cdot$ de Finetti model

Mathematics Subject Classification Primary 91B30 - Secondary 60G42 · 60K30 . $60 \mathrm{~J} 10$

\section{Introduction}

We consider the surplus process of an insurer. A classical measure for the risk are the ruin probabilities, see for example [2,10]. Even though ruin probabilities have to be seen as a technical measure, they were criticised since neither the time to ruin nor the deficit at ruin is taken into consideration. De Finetti [4], see also [6], proposed a

Hanspeter Schmidli

schmidli@math.uni-koeln.de

Katharina Bata

katharina.bata@th-koeln.de

1 Product Development and Engineering Design, TH Cologne, Gustav-Heinemann-Ufer 54, 50968 Cologne, Germany

2 Department of Mathematics, University of Cologne, Weyertal 86-90, 50931 Cologne, Germany 
more economical tool: the expected value of discounted dividends (until ruin). We will consider this, as the further measures mentioned, as a stability criterion. Dividends have, like ruin probabilities, the disadvantage that the deficit at ruin is not taken into account. This was the reason that Kulenko and Schmidli [5], motivated by a remark in [3], introduced capital injections. The insurer is not allowed to get ruined and has to make a capital injection whenever the surplus becomes negative. The measure is then the discounted value of dividends minus the penalised capital injections. The discounting rate has to be seen as a preference parameter. That is, dividends today are preferred to dividends tomorrow, and capital injections tomorrow are preferred to capital injections today. Since the capital injections are mandatory, the deficit at ruin matters. In a Markovian environment, the value function turns out to be concave, see [5]. This implies that the optimal strategy is of barrier type. That means, all capital above a barrier is paid as dividends, and no dividend is paid below the barrier.

Since capital injections can only be avoided by the price of no dividend payments, Schmidli [7-9] introduced tax payments for dividends. Capital injections lead to an exemption from tax. That is, the amount injected can later be paid as dividends without paying tax. For example in Switzerland a capital injection can be accounted as capital reserve and later be paid back as dividend without taxes. Alternatively, dividends without tax are paid at times when the surplus without dividends is at a maximum. Therefore, the tax can also be interpreted as a tax for the company and so less surplus is present to pay as dividends, see also [1]. In the continuous time models mentioned above, the optimal strategy turns out to be very simple. When an instantaneous dividend can be paid without tax, a barrier strategy with the same barrier as in the case without tax is applied. When taxes have to be paid, also a barrier strategy is applied. Unless both barriers are at zero, the second barrier is (as expected) higher than in the case without tax.

We now work on a probability space $(\Omega, \mathcal{F}, \mathbb{P})$. Let $\left\{Y_{i}\right\}$ be iid modelling the profit of an insurance portfolio in period $i$. Then the post-injection-pre-dividend surplus at time $n$ is

$$
X_{n}=X_{n-1}-U_{n-1}+Y_{n}+L_{n}, \quad X_{0}=x,
$$

where $x$ is the initial capital, $U_{n-1}$ the dividend at time $n-1$ and $L_{n}$ the capital injection at time $n$. In order that the problem is well defined and not trivial, we assume $\mathbb{P}[Y<0]>0$ and $\mathbb{E}[|Y|]<\infty$. We denote by $p_{k}:=\mathbb{P}[Y=k]$ for $k \in \mathbb{Z}$. The information available at time $n$ is given by the natural filtration of the $\left\{Y_{k}\right\}, \mathbb{F}=\left(\mathcal{F}_{n}\right)_{n \in \mathbb{N}}$. The processes $\left\{U_{n}\right\}$ and $\left\{L_{n}\right\}$ have to be adapted to $\mathbb{F}$.

For the problems below we only have to choose the dividend strategy $\left\{U_{k}\right\}$ because making an injection before it is necessary is not optimal. Since there is no advantage to pay dividends and make a capital injection at the same time, we only allow dividends such that $X_{n}-U_{n} \geq 0$. The capital injection process $\left\{L_{t}\right\}$ will then be determined as the minimal process that keeps the surplus positive. That is, $\left\{L_{t}\right\}$ is given through

$$
L_{n}^{U}=\left(X_{n-1}-U_{n-1}+Y_{n}\right)^{-},
$$


where $a^{-}:=\max \{-a, 0\}$ and $a^{+}:=\max \{a, 0\}$ denote the negative part and positive part of $a$, respectively. We aim to determine the optimal strategy and the corresponding value function. We will start with the model without tax and then deal with the model with tax. In both cases, we prove Bellman's equation and will show that the optimal strategy is of barrier type. Further, as an example we look at a model considered by de Finetti [4].

\section{The model without tax}

The results in this section follow similarly as in the model without injections, see [6, Section 1.2].

\subsection{The value function}

We denote the value of a dividend strategy $\left\{U_{t}\right\}$ by

$$
V^{U, L}(x)=\mathbb{E}\left[\sum_{k=0}^{\infty} \zeta^{k}\left(U_{k}-\eta L_{k}\right)\right],
$$

where $\eta>1$ is a penalty factor (higher administration costs for injections than for dividends) and $\zeta \in(0,1)$ is the preference factor. Choosing $\eta=1$ would lead to $X_{n}-U_{n} \equiv 0$ under the optimal strategy. We are looking for the value function

$$
V(x)=\sup _{U, L} V^{U, L}(x)=\sup _{U} V^{U, L^{U}}(x),
$$

and for the optimal strategy $\left\{U_{t}^{*}\right\}$, for which $V(x)=V^{U^{*}}(x)$.

Let $W_{n}:=\sum_{k=0}^{n}\left(U_{k}-\eta L_{k}\right)$. Then

$$
\begin{aligned}
\sum_{k=0}^{\infty} \zeta^{k}\left(U_{k}-\eta L_{k}\right) & =\sum_{k=0}^{\infty} \sum_{n=k}^{\infty}(1-\zeta) \zeta^{n}\left(U_{k}-\eta L_{k}\right) \\
& =(1-\zeta) \sum_{n=0}^{\infty} \zeta^{n} W_{n} .
\end{aligned}
$$

In particular, this implies that it cannot be optimal to inject capital before it is necessary since later injections are discounted stronger.

We start by proving some useful properties.

Lemma $1 V(x)$ has the following properties:

(i) $V(x)$ is bounded by

$$
x+\frac{\zeta\left(\mathbb{E}\left[Y^{+}\right]-\eta \mathbb{E}\left[Y^{-}\right]\right)}{1-\zeta} \leq V(x) \leq x+\frac{\zeta \mathbb{E}\left[Y^{+}\right]}{1-\zeta} .
$$


(ii) $V(x)$ is strictly increasing and concave. We have further $V(x)-V(y) \geq x-y$ for all $x \geq y \geq 0$.

\section{Proof}

(i) For the upper bound we consider the pseudo-strategy $U^{\prime}$ with $U_{0}^{\prime}=x$ and $U_{n}^{\prime}=Y_{n}^{+}$for $n \geq 1$ and $L_{n}^{\prime}=0$. Because $X_{n}^{\prime} \leq 0$, we have $W_{n}^{\prime} \geq W_{n}$ for any strategy. From (3) we get

$$
\begin{aligned}
V^{U}(x) & =\mathbb{E}\left[\sum_{n=0}^{\infty} \zeta^{n}\left(U_{n}-\eta L_{n}^{U}\right)\right] \leq \mathbb{E}\left[\sum_{n=0}^{\infty} \zeta^{n}\left(U_{n}^{\prime}-\eta L_{n}^{U^{\prime}}\right)\right] \\
& =x+\mathbb{E}\left[\sum_{n=1}^{\infty} \zeta^{n} Y_{n}^{+}\right]=x+\frac{\zeta \mathbb{E}\left[Y^{+}\right]}{1-\zeta} .
\end{aligned}
$$

Changing the injection strategy to $L_{n}^{U^{\prime}}=Y_{n}^{-}$we get an admissible strategy. The value is

$$
\begin{aligned}
V(x) & \geq V^{U^{\prime}}(x)=x+\mathbb{E}\left[\sum_{n=1}^{\infty} \zeta^{n}\left(Y_{k}^{+}-\eta Y_{k}^{-}\right)\right] \\
& =x+\frac{\zeta\left(\mathbb{E}\left[Y^{+}\right]-\eta \mathbb{E}\left[Y^{-}\right]\right)}{1-\zeta},
\end{aligned}
$$

which yields the lower bound.

(ii) For a strategy $U$ with initial capital $y$ we define a strategy $U^{\prime}$ with initial capital $x \geq y$ through $U_{0}^{\prime}=U_{0}+x-y$ and $U_{n}^{\prime}=U_{n}$ for $n \geq 1$, and $L_{n}^{U}=L_{n}^{U^{\prime}}$ for all $n$. Then $X_{n}=X_{n}^{\prime}$ for all $n \geq 1$. This causes $V(x) \geq V^{U^{\prime}}(x)=V^{U}(y)+x-y$ which leads the assertion by taking the supremum. Concavity follows as in [5].

In the following we define $V(x)=V(0)+\eta x$ for $x<0$, and we let $Y$ be a random variable with the same distribution as $Y_{k}$. The following is proved in [6, Lemma 1.1].

\section{Proposition 1 The value function fulfils Bellman's equation}

$$
\begin{aligned}
V(x) & =\sup _{u \in[0, x]}\{u+\zeta \mathbb{E}[V(x-u+Y)]\} \\
& =\sup _{u \in[0, x]}\left\{u+\zeta\left(\sum_{k=\lfloor u-x\rfloor}^{\infty} p_{k} V(x-u+k)+\sum_{k=-\infty}^{\lfloor u-x-1\rfloor} p_{k}[V(0)+\eta(x+k-u)]\right)\right\} .
\end{aligned}
$$

Note that $u$ can only take values in a compact interval. Since $V(x)$ is concave, the function $u \mapsto \mathbb{E}[V(x-u+Y)]$ is concave, too, and therefore continuous. 
Thus there is an $u(x)$ where the supremum is attained. If more than one value $u$ exists at which the supremum is attained, then by concavity the supremum is also attained at all values between. We therefore can choose $u(x)$ as the maximal value at which the supremum is attained.

\subsection{The optimal dividend strategy}

We can now characterise the optimal strategy.

Theorem 1 The function $u(x)$ has the following properties:

(i) The number $x_{0}:=\sup \{x: u(x)=0\}$ is finite; i.e., for $x$ large enough a dividend should be paid immediately.

(ii) We have $u(x)=\left(x-x_{0}\right)^{+}$; i.e., the optimal strategy is of barrier type.

(iii) $U_{n}=u\left(X_{n}\right)$ is an optimal strategy.

(iv) If $f(x)$ is a continuous function fulfilling (5) with $f(x) \leq x+\kappa$ for some $\kappa$, then $f(x)=V(x)$.

We postpone the proof to Appendix 2.

A numerical solution can be obtained recursively as described in [6, Lemma 1.4]. The conditions are fulfilled since we only have to determine values for $x \in[0, \hat{x}]$, where $\hat{x}=(1-\zeta)^{-2} \zeta(\zeta+\eta-1) \mathbb{E}\left[Y^{-}\right]$.

We next show that it suffices to consider integer initial capital only. The proof can be found in Appendix 1.1.

Lemma 2 We can choose $x_{0} \in \mathbb{N}$. In particular, if $x \in \mathbb{N}$, then $X_{n} \in \mathbb{N}$ for all $n$. Moreover, under the optimal strategy the process visits integer points only after the first injection or the first dividend.

\subsection{All surplus as dividend}

A possible strategy is to pay all positive capital as dividend and make an injection whenever the surplus is negative. This is optimal if the value function is $V(x)=x+c:=x+(1-\zeta)^{-1} \zeta\left(\mathbb{E}\left[Y^{+}\right]-\eta \mathbb{E}\left[Y^{-}\right]\right)=: f(x)$. We now look for conditions that this is the case. That is, the optimal strategy yields $X_{n}^{*}=0$. We need to show that $f(x)$ solves (5). For $\tilde{x}=x-u$, this is equivalent to

$$
\begin{aligned}
\tilde{x}+c & \geq \zeta\left(\sum_{k=-\tilde{x}}^{\infty} p_{k} f(\tilde{x}+k)+\sum_{k=-\infty}^{-\tilde{x}-1} p_{k}[c+\eta(\tilde{x}+k)]\right) \\
& =\zeta\left(\sum_{k=-\tilde{x}}^{\infty} p_{k}[\tilde{x}+k+c]+\sum_{k=-\infty}^{-\tilde{x}-1} p_{k}[c+\eta(\tilde{x}+k)]\right) .
\end{aligned}
$$


This is equivalent to

$$
\frac{1-\zeta}{\zeta}(\tilde{x}+c)+(\eta-1) \mathbb{E}\left[(\tilde{x}+Y)^{-}\right] \geq \mathbb{E}[Y]
$$

The left hand side is convex in $\tilde{x}$. For $\tilde{x}=0$ we obtain equality by the definition of $c$. The inequality is therefore fulfilled, if it is fulfilled for $\tilde{x}=1$. That is, $\frac{1-\zeta}{\zeta}(1+c)+(\eta-1) \mathbb{E}\left[(Y+1)^{-}\right] \geq \mathbb{E}[Y]$. We therefore get the condition

$$
(1-\zeta) \geq \zeta(\eta-1) \mathbb{P}[Y<0] .
$$

E.g., the condition is fulfilled if $\eta \zeta \leq 1$. Note that, in contrast to [5], the condition depends on the distribution of $Y$.

\subsection{Example: de Finetti's model}

We now consider the following distribution for $Y, \mathbb{P}[Y=1]=1-\mathbb{P}[Y=-1]=p$ introduced in [4]. We can assume (6), or equivalently $p \geq(\zeta \eta-1) /(\zeta(\eta-1))$, since otherwise we already know the solution $V(x)=x+\zeta(p-\eta(1-p)) /(1-\zeta)$ and the optimal strategy $u(x)=x$. We find

$$
\begin{aligned}
& V(x) \\
& = \begin{cases}\zeta[p V(1)+(1-p)(V(0)-\eta)], & \text { for } x=0, \\
\zeta(p V(x+1)+(1-p) V(x-1)), & \text { for } x \in\left[1, x_{0}\right], \\
V\left(x_{0}\right)+x-x_{0}, & \text { for } x \in\left[x_{0}+1, \infty\right) .\end{cases}
\end{aligned}
$$

Then

$$
V(1)=\frac{(1-(1-p) \zeta) V(0)+(1-p) \eta \zeta}{\zeta p}
$$

Further, for $n \geq 1$

$$
V(n+1)=\frac{V(n)-\zeta(1-p) V(n-1)}{\zeta p} .
$$

Thus, $V(n)=C_{1} \lambda_{1}^{n}+C_{2} \lambda_{2}^{n}$ with

$$
\lambda_{1 / 2}=\frac{\frac{1}{\zeta} \pm \sqrt{\left(\frac{1}{\zeta}\right)^{2}-4 p(1-p)}}{2 p} .
$$

Note that $0<\lambda_{2}<1<\lambda_{1}, \lambda_{1}+\lambda_{2}=1 /(p \zeta)$ and $\lambda_{1} \lambda_{2}=(1-p) / p$. The equation for $V(1)$ yields

$$
p \zeta\left(C_{1} \lambda_{1}+C_{2} \lambda_{2}\right)=(1-(1-p) \zeta)\left(C_{1}+C_{2}\right)+(1-p) \eta \zeta .
$$

This can be written as 


$$
C_{1} \lambda_{1}+C_{2} \lambda_{2}=\left(\lambda_{1}+\lambda_{2}-\lambda_{1} \lambda_{2}\right)\left(C_{1}+C_{2}\right)+\lambda_{1} \lambda_{2} \eta
$$

At $x_{0}$ we find

$$
C_{1} \lambda_{1}^{x_{0}}+C_{2} \lambda_{2}^{x_{0}}+1=V\left(x_{0}+1\right)=C_{1} \lambda_{1}^{x_{0}+1}+C_{2} \lambda_{2}^{x_{0}+1}
$$

Thus,

$$
\begin{gathered}
\left(\lambda_{1}-1\right) \lambda_{2} C_{1}-\left(1-\lambda_{2}\right) \lambda_{1} C_{2}=\eta, \\
\left(\lambda_{1}-1\right) \lambda_{1}^{x_{0}} C_{1}-\left(1-\lambda_{2}\right) \lambda_{2}^{x_{0}} C_{2}=1 .
\end{gathered}
$$

This has the solution

$$
\begin{gathered}
C_{1}=\frac{p \zeta\left(1-\lambda_{2}\right)}{1-\zeta} \frac{\lambda_{1}-\eta \lambda_{2}^{x_{0}}}{\lambda_{1}^{x_{0}+1}-\lambda_{2}^{x_{0}+1}}, \\
C_{2}=-\frac{p \zeta\left(\lambda_{1}-1\right)}{1-\zeta} \frac{\eta \lambda_{1}^{x_{0}}-\lambda_{2}}{\lambda_{1}^{x_{0}+1}-\lambda_{2}^{x_{0}+1}} .
\end{gathered}
$$

We want to maximise $V(0)=C_{1}+C_{2}$, or equivalently to maximise

$$
\frac{\eta\left(\lambda_{1}^{x_{0}}-\lambda_{2}^{x_{0}}\right)+\lambda_{1}-\lambda_{2}}{\lambda_{1}^{x_{0}+1}-\lambda_{2}^{x_{0}+1}} .
$$

The first order condition is

$$
\eta\left(\log \lambda_{1}-\log \lambda_{2}\right) \lambda_{1}^{x_{0}} \lambda_{2}^{x_{0}}=\log \lambda_{1} \lambda_{1}^{x_{0}+1}-\log \lambda_{2} \lambda_{2}^{x_{0}+1},
$$

or equivalently

$$
\lambda_{1} \log \lambda_{1} \lambda_{2}^{-x_{0}}-\lambda_{2} \log \lambda_{2} \lambda_{1}^{-x_{0}}=\eta\left(\log \lambda_{1}-\log \lambda_{2}\right) .
$$

The solution has to be found numerically.

\section{The model with tax}

\subsection{The value function}

We now introduce tax on dividends. Let $1-\delta \in(0,1)$ denote the tax rate. As in [7-9], a capital injection leads to an exemption for tax up to the injected amount. By $Z_{n}$ we denote the amount we could immediately pay as dividend without having to pay tax. That is, letting $Z_{0}=z$ be the initial exemption, $Z_{n+1}:=\left(Z_{n}-U_{n}\right)^{+}+L_{n+1}$. The value of a dividend strategy $U$ is then defined as 


$$
V^{U}(x)=\mathbb{E}\left[\sum_{k=0}^{\infty} \zeta^{k}\left(\min \left\{U_{k}, Z_{k}\right\}+\delta\left(U_{k}-Z_{k}\right)^{+}-\eta L_{k}\right)\right] .
$$

It cannot be optimal to inject more capital than needed. We therefore omit the letter $L$ in $V^{U}(x)$ because the injection strategy is determined by the dividend strategy $U$. The value function is defined as $V(x, z)=\sup _{U} V^{U}(x, z)$ where the supremum is taken over all adapted dividend strategies $U$. Because of the tax we can allow here the value $\eta=1$.

We will need below the value of the strategy if we pay all surplus as dividend. Call this reference value $v(x, z)$. We will calculate $v(x, z)$ later.

The value function has the following properties. The proof is found in the Appendix 1.2

\section{Lemma 3}

(i) $V(x, z)$ is bounded byWe now work on a pr

$$
\begin{aligned}
& \min \{x, z\}+\delta(x-z)+\zeta \frac{\delta \mathbb{E}\left[Y^{+}\right]-\eta \mathbb{E}\left[Y^{-}\right]}{1-\zeta} \leq V(x, z) \\
& \quad \leq \min \{x, z\}+\delta(x-z)+\frac{\zeta \mathbb{E}\left[Y^{+}\right]}{1-\zeta}
\end{aligned}
$$

(ii) $V(x, z)$ is strictly increasing in both variables.

(iii) $V(x, z)$ is concave in $x$.

(iv) $V(x, z)$ is Lipschitz-continuous.

A verification theorem is proved in Appendix 3.

Theorem 2 Suppose $f: \mathbb{R} \rightarrow \mathbb{R}$ is continuous and fulfils $f(x, z) \leq x+\kappa$ for all $x$ and for some $\kappa>0, f(x, z)=f(0, z-x)+\eta x$ for $x \leq 0$ and for $x \geq 0$

$$
\begin{aligned}
f(x, z)= & \sup _{0 \leq u \leq x} \min \{u, z\}+\delta(u-z)^{+} \\
& +\zeta \sum_{k=-\infty}^{\infty} p_{k} f\left(x+k-u,(z-u)^{+}\right) .
\end{aligned}
$$

Then $f(x, z)=V(x, z)$. On the other hand, $V(x, z)$ fulfils $(10)$. 


\subsection{The optimal strategy}

As in Sect. 2 it follows that an optimal strategy exist. We denote by $u(x, z)$ the optimal dividend and we choose the largest value for $u$ if the maximiser is not unique. The optimal strategy has the following properties.

\section{Theorem 3}

(i) The number $x_{1}:=\sup \{x: u(x, z)=0$ for some $z \geq 0\}$ is finite; i.e., for $x$ large enough a dividend should be paid.

(ii) For each $z$, there is $x_{0}(z)$, such that $u(x, z)=\left(x-x_{0}(z)\right)^{+}$.

Proof The first assertion follows similarly as in the proof of Theorem 1 . The second assertion follows from the concavity of $V(x, z)$ in the first argument. $Z_{t}$.

The optimal strategy is thus a barrier strategy, where the barrier may depend on

Similarly as in Lemma 2 it follows that we can restrict to integer values. We thus assume in the following that $x, z, u \in \mathbb{N}$.

\subsection{Premia of size one}

We now make the additional assumption $\mathbb{P}[Y \geq 2]=0$ and $p_{1}>0$. That is a risk model in discrete time where the monetary unit is the premium income of a period.

Remark If $p_{1}=0$, that is $\mathbb{P}[Y \leq 0]=1$, we have $V(0, z)=(1-\zeta)^{-1} \eta \zeta \mathbb{E}[Y]$. The solution can then be calculated recursively for $n \in \mathbb{N}$ from (10). If $\quad p_{0}=0$, then either $\quad V(x+1, z)=\zeta \sum_{k=-\infty}^{-1} p_{k} V(x+1+k, z) \quad$ or $V(x+1, z)=\mathbb{1}_{z \geq 1}+\delta \mathbb{1}_{z=0}+V\left(x,(z-1)^{+}\right)$. We choose the larger of the two values. If $p_{0}>0$, then either $V(x+1, z)=\left(1-\zeta p_{0}\right)^{-1} \zeta \sum_{k=-\infty}^{-1} p_{k} V(x+1+k, z)$ or $V(x+1, z)=\mathbb{1}_{z \geq 1}+\delta \mathbb{1}_{z=0}+V\left(x,(z-1)^{+}\right)$, whichever is larger.

\subsubsection{Preliminaries}

Let $\xi>1$ be the solution to the equation $\zeta \mathbb{E}\left[\xi^{Y}\right]=1$. By $f_{0}(x)$ for $x \geq 0$ denote the solution to

$$
f_{0}(x)=\zeta\left[\sum_{k=-x}^{1} p_{k} f_{0}(x+k)+\sum_{-\infty}^{-x-1} p_{k}\left(f_{0}(0)+\eta(x+k)\right)\right],
$$

where $f_{0}(0)$ is the value at zero of the model without tax. From 


$$
\begin{aligned}
& f_{0}(x+1) \\
& =\frac{1}{\zeta p_{1}}\left(f_{0}(x)-\zeta\left[\sum_{k=-x}^{0} p_{k} f_{0}(x+k)+\sum_{-\infty}^{-x-1} p_{k}\left(f_{0}(0)+\eta(x+k)\right)\right]\right),
\end{aligned}
$$

we see that the solution exists and is unique. Note that for $x \leq x_{0}+1$, where $x_{0}$ was defined in Lemma 2, $f_{0}(x)$ is the value of the model without tax.

Lemma 4 We have $f_{0}(x+1)-f_{0}(x) \geq 1$ for all $x \geq 0$.

We prove the result in Appendix 1.3.

The result shows that in the model without tax we have to choose the initial value such that the minimal increment is one. In particular, $f_{0}(x)$ is strictly increasing with $f_{0}(x) \rightarrow \infty$ as $x \rightarrow \infty$.

Let

$$
f(x, z)=f_{0}(x)-C \xi^{x-z},
$$

and choose $C$ such that $\min \{f(x+1,0)-f(x, 0): x \geq 0\}=\delta$. We let $x_{1}$ be the argument for which the minimum is attained. Note that

$$
\begin{aligned}
1-C \xi^{x_{0}}(\xi-1) & =f_{0}\left(x_{0}+1\right)-f_{0}\left(x_{0}\right)-C \xi^{x_{0}}(\xi-1) \geq \delta \\
& =f_{0}\left(x_{1}+1\right)-f_{0}\left(x_{1}\right)-C \xi^{x_{1}}(\xi-1) \geq 1-C \xi^{x_{1}}(\xi-1) .
\end{aligned}
$$

This implies that $x_{0} \leq x_{1}$. We need to verify the existence of $x_{1}$.

Lemma 5 The constants $C$ and $x_{1}$ exist.

For the proof see Appendix 1.4.

\subsubsection{The solution to the problem}

We can now solve the problem.

Theorem 4 If $\mathbb{P}[Y \geq 2]=0$ and $p_{1}>0$ then

$$
\begin{aligned}
& V(x, z) \\
& \quad= \begin{cases}f(0, z-x)+\eta x, & \text { if } x<0, \\
f(x, 0), & \text { if } 0 \leq x \leq x_{1} \text { and } z=0, \\
f\left(x_{1}, 0\right)+\delta\left(x-x_{1}\right), & \text { if } x>x_{1} \text { and } z=0, \\
f(x, z), & \text { if } 0 \leq x \leq x_{0} \text { and } z \geq 1, \\
\left.f\left(\max \left\{x_{0}, x-z\right)\right\},\left(z-x+x_{0}\right)^{+}\right)+\min \left\{x-x_{0}, z\right\}, & \text { if } x>x_{0} \text { and } z \geq 1 .\end{cases}
\end{aligned}
$$

The constant $x_{0}$ is the dividend barrier from the model without tax.

The proof is postponed to Appendix 4 . 
Theorem 4 implies that the optimal strategy is, as in [7-9], a strategy with two barriers. If an immediate dividend of size one is not taxed, the dividend barrier $x_{0}$ is used. That is, all capital above $x_{0}$ is paid as dividend. If tax has to be paid for an immediate dividend, the barrier $x_{1}$ is used. That means, if starting with an initial capital below $x_{0}$, then as long as $Z_{t}>0$ the premium is paid as dividend until the next claim occurs. Whenever $Z_{t}$ reaches zero, no dividend is paid until the surplus reaches $x_{1}$. Then the premium is paid as dividend until the next time of a claim.

Corollary 1 The optimal strategy is given as

$$
u(x, z)=\max \left\{x-x_{1}, \min \left\{\left(x-x_{0}\right)^{+}, z\right\}\right\} .
$$

\subsubsection{All surplus as dividend}

We next consider the strategy $u(x, z)=x$, that is, to pay all surplus as dividend.

Lemma 6 The value of the strategy paying all surplus as dividends has the value

$$
\min \{x, z\}+\delta(x-z)^{+}+\frac{\zeta}{1-\zeta}\left(p_{1}-\eta \mathbb{E}\left[Y^{-}\right]\right)-\xi^{-(z-x)^{+}} \frac{1-\delta}{\xi-1} .
$$

Proof The value of the dividend at zero is $\min \{x, z\}+\delta(x-z)^{+}$. Thus we can assume $x=0$. The second term is the value of the dividends if no tax had to be paid. We therefore need to calculate the value of the dividends for which tax is paid. Note that $S_{n}=\sum_{k=1}^{n}\left(U_{k}-L_{k}\right)$. It is easy to see that $\mathcal{T}_{n}=\left(\max _{k \leq n} S_{k}-z\right)^{+}$. The discounting of the first time point with $Z_{n}=0$ is $\mathbb{E}\left[\zeta^{\sigma_{z}}\right]$, where $\sigma_{z}=\inf \left\{n \geq 0: S_{n}=z\right\}$. We get $\mathbb{E}\left[\zeta^{\sigma_{z}}\right]=\mathbb{E}^{*}\left[\xi^{-S_{\sigma_{z}}}\right]=\xi^{-z}$, where the measure $\mathbb{P}^{*}$ is defined in Appendix 1.4. For $z=0$, let $v_{0}=\mathbb{E}\left[\sum_{n=1}^{\infty} \zeta^{n} \Delta \mathcal{T}_{n}\right]$ be the value of the dividends. Then $Z_{1}=Y_{1}^{-}$and

$$
v_{0}=\zeta\left[p_{1}\left(1+v_{0}\right)+\left(\sum_{k=-\infty}^{0} p_{k} \xi^{k} v_{0}\right)\right]=v_{0}+p_{1} \zeta\left(1+v_{0}-\xi v_{0}\right) \text {. }
$$

Thus $v_{0}=(\xi-1)^{-1}$. This yields the last term.

We next look for conditions under which it is optimal to pay all surplus as dividend. That is, we have to verify that (10) is fulfilled. For simplicity, we let $f(x, z)=\min \{x, z\}+\delta(x-z)^{+}+\kappa_{1}-\kappa_{2} \xi^{-(z-x)^{+}}$for $x \geq 0$, where $\kappa_{1}$ and $\kappa_{2}$ are the constants from Lemma 6 , and $f(x, z)=f(0, z-x)+\eta x$ for $x<0$. Then for $z=0$, 


$$
\begin{aligned}
g(x)= & f(x, 0)-\zeta \mathbb{E}[f(x+Y, 0)]=\delta x+\kappa_{1}-\kappa_{2} \\
& -\zeta \sum_{k=-x}^{1} p_{k}\left(\delta(x+k)+\kappa_{1}-\kappa_{2}\right) \\
& -\zeta \sum_{k=-\infty}^{-x-1} p_{k}\left(\kappa_{1}-\kappa_{2} \xi^{x+k}+\eta(x+k)\right) \\
= & \delta\left(x-\zeta \mathbb{E}\left[(Y+x)^{+}\right]\right)+\eta \zeta \mathbb{E}\left[(Y+x)^{-}\right]+(1-\zeta) \kappa_{1} \\
& \quad-\kappa_{2}\left(1-\zeta \mathbb{E}\left[\xi^{-(Y+x)^{-}}\right]\right) .
\end{aligned}
$$

Then $g(0)=0$ and

$$
\begin{aligned}
g(x+1)-g(x) & =\delta(1-\zeta \mathbb{P}[Y \geq-x])-\eta \zeta \mathbb{P}[Y<-x]+\zeta \kappa_{2}(\xi-1) \mathbb{E}\left[\xi^{Y+x} \mathbb{1}_{Y<-x}\right] \\
& =\delta-\zeta-(\eta-1) \zeta \mathbb{P}[Y<-x]+\zeta(1-\delta) \mathbb{E}\left[\xi^{-(Y+x)^{-}}\right] .
\end{aligned}
$$

This function is increasing in $x$. Therefore, (10) is fulfilled if $g(1)=g(1)-g(0) \geq 0$. That is

$$
\begin{aligned}
0 \leq & \delta\left(1-\zeta\left(p_{1}+p_{0}\right)\right)-\eta \zeta\left(1-p_{1}-p_{0}\right) \\
& \quad+(1-\delta)\left(1-\zeta\left(p_{0}+p_{1} \xi\right)\right) \\
= & 1-\zeta\left[\eta+(\xi(1-\delta)-\eta+\delta) p_{1}-(\eta-1) p_{0}\right] .
\end{aligned}
$$

For $z \geq 1$ and $x<z$

$$
\begin{aligned}
g(x, z)= & f(x, z)-\zeta \mathbb{E}[f(x+Y, z)]=x+\kappa_{1}-\kappa_{2} \xi^{x-z} \\
& -\zeta \sum_{k=-x}^{1} p_{k}\left(x+k+\kappa_{1}-\kappa_{2} \xi^{k+x-z}\right) \\
& -\zeta \sum_{k=-\infty}^{-x-1} p_{k}\left(\kappa_{1}-\kappa_{2} \xi^{x+k-z}+\eta(x+k)\right) \\
= & x-\zeta \mathbb{E}\left[(Y+x)^{+}\right]+\eta \zeta \mathbb{E}\left[(Y+x)^{-}\right]+(1-\zeta) \kappa_{1},
\end{aligned}
$$

where we used the definition of $\xi$. Analogous to the case $z=0$ it follows that (10) is fulfilled if $g(1, z) \geq 0$ or

$$
1-\zeta\left[\eta-(\eta-1)\left(p_{0}+p_{1}\right)\right] \geq 0,
$$

which is always fulfilled if the condition for $z=0$ is fulfilled.

Suppose now $x \geq z$. Then 


$$
\begin{aligned}
g(x, z)= & f(x, z)-\zeta \mathbb{E}[f(x+Y, z)]=z+\delta(x-z)+\kappa_{1}-\kappa_{2} \\
& -\zeta\left[\sum_{k=z-x}^{1} p_{k}\left(z+\delta(x+k-z)+\kappa_{1}-\kappa_{2}\right)\right. \\
& +\sum_{k=-x}^{z-x-1} p_{k}\left(x+k+\kappa_{1}-\kappa_{2} \xi^{x+k-z}\right) \\
& \left.+\sum_{k=-\infty}^{-x-1} p_{k}\left(\kappa_{1}-\kappa_{2} \xi^{x+k-z}+\eta(x+k)\right)\right]
\end{aligned}
$$

The condition for $g(x, 0) \geq 0$ was obtained above. Further,

$$
\begin{aligned}
& g(x+1, z+1)-g(x, z) \\
& \quad=1-\zeta-\zeta(\eta-1) \mathbb{P}[Y<-x] .
\end{aligned}
$$

This shows that $g(x+z, z)$ is increasing in $z$ and therefore (10) is fulfilled if (12) holds.

\subsection{De Finetti's example}

Suppose again $\mathbb{P}[Y=1]=1-\mathbb{P}[Y=-1]=p$. We find $\xi=\lambda_{1}$, where $\lambda_{k}$ is defined in Sect. 2.4. Condition (12) reads then

$$
\zeta[\eta-(\eta-\delta-\xi(1-\delta)) p] \leq 1,
$$

or equivalently,

$$
p \geq \frac{\eta \zeta-1}{\zeta(\eta-\delta-\xi(1-\delta))}
$$

We therefore assume in the sequel that

$$
0<p<\frac{\eta \zeta-1}{\zeta(\eta-\delta-\xi(1-\delta))}
$$

Suppose first $p \geq(\zeta \eta-1) /(\zeta(\eta-1))$, that is $x_{0}=0$. Then $f_{0}(x)=C_{1} \lambda_{1}^{x}+C_{2} \lambda_{2}^{x}$ with

$$
\begin{aligned}
C_{1}+C_{2} & =(1-\zeta)^{-1} \zeta(p-\eta(1-p))=f(0) \\
& =\zeta\left[p\left(C_{1} \lambda_{1}+C_{2} \lambda_{2}\right)+(1-p)\left(C_{1}+C_{2}-\eta\right)\right] .
\end{aligned}
$$

Equivalently,

$$
\begin{aligned}
\left(\lambda_{1}-1\right) C_{1}-\left(1-\lambda_{2}\right) C_{2} & =1 \\
(1-\zeta) C_{1}+(1-\zeta) C_{2} & =\zeta(p-\eta(1-p))
\end{aligned}
$$

Thus, 


$$
\begin{aligned}
C_{1} & =\frac{1-\zeta+\zeta\left(1-\lambda_{2}\right)(p-(1-p) \eta)}{(1-\zeta)\left(\lambda_{1}-\lambda_{2}\right)}, \\
C_{2} & =\frac{\zeta\left(\lambda_{1}-1\right)(p-\eta(1-p))+\zeta-1}{(1-\zeta)\left(\lambda_{1}-\lambda_{2}\right)} .
\end{aligned}
$$

If $p<(\zeta \eta-1) /(\zeta(\eta-1))$, then $f_{0}(x)=C_{1} \lambda_{1}^{x}+C_{2} \lambda_{2}^{x}$ with the constants determined in the case without tax.

We look for

$$
\inf \left\{\left(C_{1}-\tilde{C}\right) \lambda_{1}^{\tilde{x}_{1}}+C_{2} \lambda_{2}^{\tilde{x}_{1}}\right\}=\delta .
$$

The first order condition for the minimum is

$$
\left(C_{1}-\tilde{C}\right) \log \lambda_{1} \lambda_{1}^{\tilde{x}_{1}}+C_{2} \log \lambda_{2} \lambda_{2}^{\tilde{x}_{1}}=0,
$$

which is indeed a minimum if $C_{2}>0$ or equivalently, $C_{1}>\tilde{C}$. This is

$$
\left(\frac{\lambda_{1}}{\lambda_{2}}\right)^{\tilde{x}_{1}}=\frac{-C_{2} \log \lambda_{2}}{\left(C_{1}-\tilde{C}\right) \log \lambda_{1}} .
$$

Thus,

$$
C_{2}\left(\frac{-\log \lambda_{2}}{\log \lambda_{1}}+1\right) \lambda_{2}^{\tilde{x}_{1}}=\delta .
$$

From the latter equation, $\tilde{C}$ can be determined. If $\tilde{C}$ is known, also $\tilde{x}_{1}$ can be determined. Since $x_{1} \in \mathbb{N}$, we have to choose $\left\lfloor\tilde{x}_{1}\right\rfloor$ or $\left\lfloor\tilde{x}_{1}+1\right\rfloor$, and then to calculate $C$ corresponding to $x_{1}$.

\section{Conclusion}

We considered a risk model in discrete time where dividends may be paid and capital has to be injected in order to keep the surplus positive. We determined the optimal dividend strategy in order to maximise the expected discounted value of dividends minus penalised injections both for the case without and with tax. In the problem with tax and premia of size one we could determine explicitly the form of the optimal strategy. The value function considered has to be interpreted as a stability measure. The dividend payments are a measure for the profits, the injections a measure for the losses. The penalising factor reflects that large losses cause administrative action and therefore lead to additional costs. Further, the choice of the penalising factor gives the possibility to weight the profits and the losses differently. This technical tool may be used to measure the influence of strategic decisions on the profit and the risk. The dividend barriers give a hint how large fluctuation reserves are reasonable to be kept. Too low fluctuation reserves means one needs to raise money for large losses. Too large reserves means that one loses money because of regulative restrictions for investments. Tax payments occur 
at times where the process without dividends and injections reaches a new maximum. In practice, a company has then to pay taxes, or the share holders require dividends. Taking this into account, reserves should not increase too much in such a situation.

Acknowledgements Open Access funding provided by Projekt DEAL.

Open Access This article is licensed under a Creative Commons Attribution 4.0 International License, which permits use, sharing, adaptation, distribution and reproduction in any medium or format, as long as you give appropriate credit to the original author(s) and the source, provide a link to the Creative Commons licence, and indicate if changes were made. The images or other third party material in this article are included in the article's Creative Commons licence, unless indicated otherwise in a credit line to the material. If material is not included in the article's Creative Commons licence and your intended use is not permitted by statutory regulation or exceeds the permitted use, you will need to obtain permission directly from the copyright holder. To view a copy of this licence, visit http://creativecommons.org/licen ses/by/4.0/.

\section{Appendix 1: Proofs of Lemmata}

\section{Proof of Lemma 2}

Suppose $x_{0} \notin \mathbb{N}$. Let $\left\lfloor x_{0}\right\rfloor \leq x \leq x_{0}$. Let $\mathbb{E}_{x}$ denote the law with initial capital $x$. Let $\tau=\inf \left\{n>0: X_{n}-L_{n} \notin\left[0, x_{0}\right]\right\}$. Note that $\tau$ does not depend on $x$. Then

$$
\begin{aligned}
V(x) & =\mathbb{E}_{x}\left[\left(V\left(X_{\tau}\right)-\eta L_{\tau}\right) \zeta^{\tau}\right] \\
& =\mathbb{E}_{x_{0}}\left[\left(V\left(X_{\tau}\right)-\eta\left(L_{\tau}+\left(x_{0}-x\right)\right)\right) \zeta^{\tau}\right] .
\end{aligned}
$$

The difference between the first dividends is also $x_{0}-x$ if it is paid at time $\tau$. After time $\tau$ the dividend and injection processes coincide. Thus,

$$
V\left(x_{0}\right)-V(x)=\left(x_{0}-x\right) \mathbb{E}_{x_{0}}\left[\left(\mathbb{1}_{X_{\tau}>x_{0}}+\eta \mathbb{1}_{X_{\tau}=0}\right) \zeta^{\tau}\right] .
$$

We conclude that $\mathbb{E}_{x_{0}}\left[\left(\mathbb{1}_{X_{\tau}>x_{0}}+\eta \mathbb{1}_{X_{\tau}=0}\right) \zeta^{\tau}\right] \geq 1$. For $x_{0}<x<\left\lfloor x_{0}+1\right\rfloor$, we follow the (maybe not optimal) strategy not paying a dividend before $\tau$ and then to follow the optimal strategy. The we obtain analogously, $\mathbb{E}_{x_{0}}\left[\left(\mathbb{1}_{X_{\tau}>x_{0}}+\eta \mathbb{1}_{X_{\tau}=0}\right) \zeta^{\tau}\right] \leq 1$ because $V(x)-V\left(x_{0}\right)=x-x_{0}$. Thus we have equality and $V\left(x_{0}\right)=V\left(\left\lfloor x_{0}\right\rfloor\right)+x_{0}-\left\lfloor x_{0}\right\rfloor$. Thus we can choose $x_{0} \in \mathbb{N}$.

\section{Proof of Lemma 3}

(i) The lower bound is the value of the strategy if all surplus is paid as dividend and tax has to be paid for all the dividends except for the amount $z$.

For the upper bound note that 


$$
\begin{aligned}
& \sum_{k=0}^{\infty} \zeta^{k}\left(\min \left\{U_{k}, Z_{k}\right\}+\delta\left(U_{k}-Z_{k}\right)^{+}-\eta L_{k}\right) \\
& \quad=(1-\zeta) \sum_{\ell=0}^{\infty} \zeta^{\ell} \sum_{k=0}^{\ell} \min \left\{U_{k}, Z_{k}\right\}+\delta\left(U_{k}-Z_{k}\right)^{+}-\eta L_{k} .
\end{aligned}
$$

The right hand side can be made maximal if all capital is paid as dividend but no injections are made, and no tax has to be paid for all dividends after the first dividend.

(ii) This is trivial.

(iii) Let $x<y$ and $v=(1-\alpha) x+\alpha y$ for an $\alpha \in(0,1)$. Consider a strategy $\left\{U_{n}^{x}\right\}$ and $\left\{U_{n}^{y}\right\}$ for initial capital $x$ and $y$, respectively, with corresponding injection strategies $\left\{L_{n}^{x}\right\}$ and $\left\{L_{n}^{y}\right\}$, respectively. Then the strategy $U_{n}^{v}=(1-\alpha) U_{n}^{x}+\alpha U_{n}^{y}$ and $L_{n}^{v}=(1-\alpha) L_{n}^{x}+\alpha L_{n}^{y}$ is admissible for initial capital $v$. Let $\mathcal{T}_{n}=\sum_{k=0}^{n}\left(U_{k}-Z_{k}\right)^{+}$be the accumulated dividends for which tax is paid. Then

$$
\sum_{k=0}^{n} U_{k}+Z_{n+1}=z+\sum_{k=1}^{n+1} L_{k}+\mathcal{T}_{n}
$$

Note that this implies

$$
\mathcal{T}_{n} \geq \sum_{k=0}^{n} U_{k}-z-\sum_{k=1}^{n+1} L_{k} .
$$

$\mathcal{T}_{n}^{\mathcal{D}}$ only increases at points where $U_{n}^{v}>Z_{n}^{v}$. At such a point we get $Z_{n+1}^{v}=0$. Thus

$$
\begin{aligned}
\mathcal{T}_{n} & =\sum_{k=0}^{n} U_{k}^{v}-z-\sum_{k=1}^{n+1} L_{k}^{v} \\
& =(1-\alpha)\left[\sum_{k=0}^{n} U_{k}^{x}-z-\sum_{k=1}^{n+1} L_{k}^{x}\right]+\alpha\left[\sum_{k=0}^{n} U_{k}^{y}-z-\sum_{k=1}^{n+1} L_{k}^{y}\right] \\
& \leq(1-\alpha) \mathcal{T}_{n}^{\mathcal{x}}+\alpha \mathcal{T}_{n}^{y} .
\end{aligned}
$$

Concavity follows now similarly as in [5].

(iv) It suffices to show Lipschitz continuity in each of the variables. Let $x<y$ and $z \geq 0$. Choose $\epsilon>0$. There is a strategy $U$, such that $V(y, z)<V^{U}(y, z)+\epsilon$. Using the strategy $U$ for initial capital $x$, additional injections may have to be made (or less dividends may be paid if the surplus would become negative otherwise). This shows that $V(x, z) \geq V^{U}(x, z) \geq V^{U}(y, z)-\eta(y-x)>V(y, z)-\eta(y-x)-\epsilon$. Since $\epsilon$ was arbitrary, $V(y, z)-V(x, z) \leq \eta(y-x)$. 
Let $x \geq 0$ and $z_{1}<z_{2}$. Similarly, as for the first argument we obtain $V\left(x, z_{2}\right)-V\left(x, z_{1}\right) \leq(1-\delta)\left(z_{2}-z_{1}\right)$, since using the same strategy may lead to additional tax payments.

\section{Proof of Lemma 4}

Suppose the assertion does not hold. Let $x_{2}$ be a point where $\gamma=f_{0}\left(x_{2}+1\right)-f_{0}\left(x_{2}\right)<1$. We consider now the model without tax. Note that $x_{2}>x_{0}$ by the results of Section 2. Consider the strategy $U_{n}=\mathbb{1}_{X_{n}=x_{2}+1}$ and suppose $x=X_{0} \leq x_{0}+1$. Then

$$
\begin{aligned}
\mathbb{E} & {\left[\zeta f_{0}\left(X_{k}+L_{k+1}-U_{k}+Y_{k+1}\right) \mid \mathcal{F}_{k}\right] } \\
& =\mathbb{E}\left[\zeta\left(f_{0}\left(X_{k}-U_{k}+Y_{k+1}\right)+\eta L_{k+1}\right) \mid \mathcal{F}_{k}\right] \\
& =f_{0}\left(X_{k}\right)-\gamma \mathbb{1}_{X_{k}=x_{2}+1}+\eta \zeta \mathbb{E}\left[L_{k+1} \mid \mathcal{F}_{k}\right] \\
& =f_{0}\left(X_{k}\right)-\gamma U_{k}+\eta \zeta \mathbb{E}\left[L_{k+1} \mid \mathcal{F}_{k}\right],
\end{aligned}
$$

Thus

$$
\begin{aligned}
f_{0}(x)=\mathbb{E} & {\left[\zeta^{n} f_{0}\left(X_{n}\right)-\sum_{k=0}^{n-1}\left\{\zeta^{k+1} f_{0}\left(X_{k+1}\right)-\zeta^{k} f_{0}\left(X_{k}\right)\right\}\right] } \\
=\mathbb{E} & {\left[\zeta^{n} f_{0}\left(X_{n}\right)-\sum_{k=0}^{n-1} \zeta^{k}\left\{\zeta f_{0}\left(X_{k}+L_{k+1}-U_{k}+Y_{k+1}\right)\right.\right.} \\
& \left.\left.-f_{0}\left(X_{k}\right)\right\}\right] \\
=\mathbb{E} & {\left[\zeta^{n} f_{0}\left(X_{n}\right)+\sum_{k=0}^{n-1} \gamma \zeta^{k} U_{k}-\eta \zeta^{k+1} L_{k+1}\right] . }
\end{aligned}
$$

Because $\left\{X_{n}\right\}$ is bounded by $x_{2}+1$, we can let $n \rightarrow \infty$ obtaining

$$
f_{0}(x)=\mathbb{E}\left[\sum_{k=0}^{\infty} \zeta^{k} \gamma U_{k}-\eta \zeta^{k+1} L_{k+1}\right] \text {. }
$$

This is the value of the proposed strategy but with all dividends taxed with the rate $1-\gamma$. Thus, the value function of the model without tax is strictly larger than $f_{0}(x)$, contradicting the fact that $f_{0}(x)$ is the value function for $x \leq x_{0}+1$.

\section{Proof of Lemma 5}

Let $a>x \geq 0$. We consider the process $S_{n}=x+\sum_{k=1}^{n} Y_{k}$ and define the stopping times $\tau^{a}=\inf \left\{n>0: S_{n}=a\right\} \quad$ and $\tau_{-}=\inf \left\{n>0: S_{n}<0\right\}$. We 
define the measure $\mathbb{P}^{*}[A]=\zeta^{n} \mathbb{E}\left[\xi^{S_{n}-x} \mathbb{1}_{A}\right]$ on $\mathcal{F}_{n}$, see $[10$, Ch. 8]. We have $\mathbb{E}^{*}[Y]=\zeta \mathbb{E}\left[Y \xi^{Y}\right]>0$ because of the concavity of $x \mapsto \mathbb{E}\left[\mathrm{e}^{x Y}\right]$. As above, we find

$$
f_{0}(x)=\mathbb{E}\left[\zeta^{\tau^{a} \wedge \tau_{-}} f_{0}\left(S_{\tau^{a} \wedge \tau_{-}}\right)\right]=\mathbb{E}^{*}\left[\xi^{x-S_{\tau^{a} \wedge \tau_{-}}} f_{0}\left(S_{\tau^{a} \wedge \tau_{-}}\right)\right] .
$$

This implies

$$
f_{0}(x) \xi^{-x}=\mathbb{E}^{*}\left[\xi^{-S_{\tau_{-}}} f_{0}\left(S_{\tau_{-}}\right) \mathbb{1}_{\tau_{-}<\tau^{a}}\right]+f_{0}(a) \xi^{-a} \mathbb{P}^{*}\left[\tau^{a}<\tau_{-}\right] .
$$

As $a \rightarrow \infty, \mathbb{P}^{*}\left[\tau^{a}<\tau_{-}\right]$converges to the survival probability under $\mathbb{P}^{*}$. The other term

$$
\mathbb{E}^{*}\left[\xi^{-S_{\tau_{-}}} f_{0}\left(S_{\tau_{-}}\right) \mathbb{1}_{\tau_{-}<\tau^{a}}\right]=\mathbb{E}\left[\zeta^{\tau_{-}} f_{0}\left(S_{\tau_{-}}\right) \mathbb{1}_{\tau_{-}<\tau^{a}}\right] \xi^{-x}
$$

converges to the Gerber-Shiu function $\mathbb{E}\left[\zeta^{\tau_{-}} f_{0}\left(S_{\tau_{-}}\right) \mathbb{1}_{\tau_{-}<\infty}\right] \zeta^{-x}$. This implies that the second term converges. Since the net profit condition is fulfilled under $\mathbb{P}^{*}$, that is $\mathbb{P}^{*}\left[\tau_{-}=\infty\right]>0$, the limit of $f_{0}(a) \xi^{-a}$ must be finite. We claim that the limit is nonzero. If the latter was not the case, we had

$$
f_{0}(x)=\mathbb{E}\left[\zeta^{\tau}-f_{0}\left(S_{\tau_{-}}\right) \mathbb{1}_{\tau_{-}<\tau^{a}}\right] \leq \max \left\{f_{0}(0), 0\right\},
$$

which cannot hold for all $x$ by Lemma 4 . Thus, $\lim _{x \rightarrow \infty} f_{0}(x) \xi^{-x}>0$. This shows the assertion.

\section{Appendix 2: Proof of Theorem 1}

(i) If $u(x)=0$ we get from (4)

$$
\begin{aligned}
x+\zeta \frac{\mathbb{E}\left[Y^{+}\right]-\eta \mathbb{E}\left[Y^{-}\right]}{1-\zeta} & \leq V(x)=\zeta \sum_{j=-\infty}^{\infty} p_{j} V(x+j) \\
& \leq \zeta \sum_{j=-\infty}^{\infty} p_{j}\left(x+j+\frac{\mathbb{E}\left[Y^{+}\right] \zeta}{1-\zeta}\right) \\
& =\zeta x+\zeta\left(\mathbb{E}[Y]+\frac{\mathbb{E}\left[Y^{+}\right] \zeta}{1-\zeta}\right) \\
& =\zeta x+\zeta \frac{\mathbb{E}[Y]+\zeta \mathbb{E}\left[Y^{-}\right]}{1-\zeta},
\end{aligned}
$$

where we used that the upper bound also holds for $x<0$. This implies

$$
x \leq \frac{\zeta(\zeta+\eta-1)}{(1-\zeta)^{2}} \mathbb{E}\left[Y^{-}\right]
$$

Thus $u(y) \neq 0$ for $y$ larger than the right hand side.

(ii) Let $\tilde{x}=x-u(x)$. Then 


$$
\begin{aligned}
u(x) & =x-\tilde{x} \leq V(x)-V(\tilde{x}) \leq V(x)-\zeta \mathbb{E}[V(\tilde{x}+Y)] \\
& =V(x)-\zeta \mathbb{E}[V(x-u(x)+Y)]=u(x) .
\end{aligned}
$$

Thus $V(\tilde{x})=\zeta \mathbb{E}[V(\tilde{x}+Y)]$ and $V(x)-V(\tilde{x})=u(x)$. Note that

$$
\begin{aligned}
V(x) & =u(x)+\zeta \mathbb{E}[V(\tilde{x}+Y)] \\
& =u(x)+u(\tilde{x})+\zeta \mathbb{E}[V(x-u(x)-u(\tilde{x})+Y)] .
\end{aligned}
$$

Because $u(x)$ is the largest value at which the supremum is taken, we have $u(\tilde{x})=0$. Suppose that $u(x)>0$ and choose $y>x$. We have by the concavity

$$
V(\tilde{x})+x-\tilde{x}=V(x) \geq \frac{x-\tilde{x}}{y-\tilde{x}} V(y)+\frac{y-x}{y-\tilde{x}} V(\tilde{x}),
$$

or equivalently, $V(y) \leq y-\tilde{x}+V(\tilde{x})$. By Lemma 1 , we have equality. This implies that $u(y) \geq y-\tilde{x}$. Now,

$$
\begin{aligned}
& u(y)+V(y-u(y))=V(y)=y-\tilde{x}+V(\tilde{x}) \\
& \quad \geq y-\tilde{x}+(\tilde{x}-(y-u(y)))+V(y-u(y))=u(y)+V(y-u(y)) .
\end{aligned}
$$

Thus $V(\tilde{x})=\tilde{x}-(y-u(y))+V(y-u(y))$. Since $u(\tilde{x})=0 \quad$ we find $u(y)=y-\tilde{x}$. This shows that there is $\tilde{x}_{0}$ such that $u(x)=\left(x-\tilde{x}_{0}\right)^{+}$. By the definition of $x_{0}$, we get $\tilde{x}_{0}=x_{0}$.

(iii) follows like the corresponding statement in [6, Theorem 1.10].

(iv) Let $\tilde{u}(x)$ be the optimiser if we replace $V$ by $f$. The considerations above apply also to $f$ implying that $\tilde{u}(x)$ exists. As in [6, Cor. 1.3] it follows that $f(x)=V(x)$.

\section{Appendix 3: Proof of Theorem 2}

That $V(x, z)$ fulfils equation (10) is proved in [6].

Let $\left\{U_{n}\right\}$ be an arbitrary strategy. Then

$$
\begin{aligned}
\zeta^{n} f\left(X_{n}, Z_{n}\right)-f(x, z)=\sum_{k=0}^{n-1}\left[\zeta^{k+1} f\left(X_{k+1}, Z_{k+1}\right)-\zeta^{k} f\left(X_{k}, Z_{k}\right)\right] \\
=\sum_{k=0}^{n-1} \zeta^{k}\left[\zeta f\left(X_{k}+L_{k+1}-U_{k}+Y_{k+1},\left(Z_{k}-U_{k}\right)^{+}+L_{k+1}\right)-f\left(X_{k}, Z_{k}\right)\right] .
\end{aligned}
$$

Conditioning on the information $\mathcal{F}_{k}$ up to time $k$

$$
\begin{array}{r}
\mathbb{E}\left[\zeta f\left(X_{k}+L_{k+1}-U_{k}+Y_{k+1},\left(Z_{k}-U_{k}\right)^{+}+L_{k+1}\right) \mid \mathcal{F}_{k}\right] \\
\quad=\mathbb{E}\left[\zeta f\left(X_{k}-U_{k}+Y_{k+1},\left(Z_{k}-U_{k}\right)^{+}\right)+\eta L_{k+1} \mid \mathcal{F}_{k}\right],
\end{array}
$$

because $L_{k+1}$ is only non-zero if $X_{k}-U_{k}+Y_{k+1}<0$. Further, by (10), 


$$
\mathbb{E}\left[\zeta f\left(X_{k}-U_{k}+Y_{k+1},\left(Z_{k}-U_{k}\right)^{+}\right) \mid \mathcal{F}_{k}\right] \leq f\left(X_{k}, Z_{k}\right)-\min \left\{U_{k}, Z_{k}\right\}-\delta\left(U_{k}-Z_{k}\right)^{+} .
$$

Thus

$$
\begin{aligned}
f(x, z) \geq & \mathbb{E}\left[\zeta^{n} f\left(X_{n}, Z_{n}\right)\right. \\
& \left.+\sum_{k=0}^{n-1} \zeta^{k}\left(\min \left\{U_{k}, Z_{k}\right\}+\delta\left(U_{k}-Z_{k}\right)^{+}-\zeta \eta L_{k+1}\right)\right] .
\end{aligned}
$$

We have $X_{n} \leq x+\sum_{k=1}^{n} Y_{k}^{+}$. Thus $\zeta^{n} \mathbb{E}\left[f\left(X_{n}, Z_{n}\right)\right] \leq \zeta^{n}\left(x+n \mathbb{E}\left[Y^{+}\right]+\kappa\right)$ tends to zero. From $U_{n} \leq X_{n}$ and $\sum_{k=1}^{n} L_{k} \leq \sum_{k=1}^{n} Y_{k}^{-}$we get by bounded convergence $f(x, z) \geq V(x, z)$.

Since $f$ is continuous, there exists $u(x)$, such that

$$
\begin{aligned}
\min & \{u(x), z\}+\delta(u(x)-z)^{+} \\
& +\zeta \sum_{k=-\infty}^{\infty} p_{k} f\left(x-u(x),(z-u(x))^{+}\right) \\
= & \sup _{0 \leq u \leq x} \min \{u, z\}+\delta(u-z)^{+}+\zeta \sum_{k=-\infty}^{\infty} p_{k} f\left(x-u,(z-u)^{+}\right) .
\end{aligned}
$$

Repeating the above calculations with $U_{n}=u\left(X_{n}\right)$, the inequality becomes an equality, implying $f(x, z)=V^{U}(x, z)$. Thus $f(x, z) \leq V(x, z)$, which proves the assertion.

\section{Appendix 4: Proof of Theorem 4}

We have to verify Bellman's equation. We make the additional assumption that $f(x+1,0)-f(x, 0)$ is decreasing for $x \leq x_{1}$. Note that this also holds for $x<0$ with $f(x, 0)=f(0,-x)+\eta x$.

If $z=0$ and $x \leq x_{1}$, then

$$
\begin{aligned}
f(x, 0) & =f_{0}(x)-C \xi^{x} \\
& =\zeta\left[\sum_{k=-x}^{1} p_{k}\left(f_{0}(x+k)-C \xi^{x+k}\right)+\sum_{k=-\infty}^{-x-1} p_{k}\left(f_{0}(0)+\eta(x+k)-C \xi^{x+k}\right)\right] .
\end{aligned}
$$

where we used that $f_{0}(x)$ solves $(11)$ and that $\zeta \mathbb{E}\left[\xi^{Y}\right]=1$. The above equation also holds with $x$ replaced by $x-u$. Since $\delta u+f(x-u, 0) \leq f(x, 0)$ by the definition of $x_{1}$, Bellman's equation holds in this case.

If $z \geq 1$ and $0 \leq x \leq x_{0}$, then as above 


$$
\begin{aligned}
f(x, z)= & f_{0}(x)-C \xi^{x-z} \\
= & \zeta\left[\sum_{k=-x}^{1} p_{k}\left(f_{0}(x+k)-C \xi^{x+k-z}\right)\right. \\
& \left.+\sum_{k=-\infty}^{-x-1} p_{k}\left(f_{0}(0)+\eta(x+k)-C \xi^{x+k-z}\right)\right] .
\end{aligned}
$$

The equation also holds for $x$ replaced by $x-u$ and $z$ replaced by $(z-u)^{+}$. For $u \leq z$,

$$
u+f(x-u, z-u)=u+f_{0}(x-u)-C \xi^{x-z} \leq f_{0}(x)-C \xi^{x-z}
$$

by Lemma 4 . If $z<u \leq x$, then

$$
z+\delta(u-z)+f(x-u, 0) \leq z+f(x-z, 0) \leq f(x, z),
$$

where we used the inequality in the case $z=0$ and the inequality in the case $u \leq z$. This shows (10) in that case.

If $z=0$ and $x>x_{1}$, then using $f\left(x_{1}\right)-f(v) \geq \delta v$ for $v \leq x_{1}$,

$$
\begin{aligned}
f\left(x_{1}, 0\right) & +\delta\left(x-x_{1}\right)-\zeta \sum_{k=x_{1}-x}^{1} p_{k}\left(f\left(x_{1}, 0\right)+\delta\left(x+k-x_{1}\right)\right) \\
& -\zeta \sum_{k=-x}^{x_{1}-x-1} p_{k} f(x+k, 0) \\
& -\zeta \sum_{-\infty}^{-x-1} p_{k}(f(0,-x-k)+\eta(x+k)) \\
= & (1-\zeta)\left[f\left(x_{1}, 0\right)+\delta\left(x-x_{1}\right)\right]-\zeta \delta \mathbb{E}[Y] \\
& +\zeta \sum_{k=-x}^{x_{1}-x-1} p_{k}\left(f\left(x_{1}, 0\right)-\delta x_{1}-\{f(x+k, 0)-\delta(x+k)\}\right) \\
& +\zeta \sum_{-\infty}^{-x-1} p_{k}\left(f\left(x_{1}, 0\right)-\delta x_{1}-\{f(0,-x-k)\right. \\
& +(\eta-\delta)(x+k)\}) \\
= & (1-\zeta)\left[f\left(x_{1}, 0\right)+\delta\left(x-x_{1}\right)\right]-\zeta \delta \mathbb{E}[Y] \\
& +\zeta \sum_{k=-\infty}^{x_{1}-x-1} p_{k}\left(f\left(x_{1}, 0\right)-\delta x_{1}-\{f(x+k, 0)-\delta(x+k)\}\right) .
\end{aligned}
$$

This is increasing in $x$ under our additional assumption. For $x=x_{1}$ we get zero. Therefore, the above expression is positive for all $x$. The inequality remains true if we replace $x$ with $x-u$ for $u \leq x-x_{1}$ and equality holds for $u=x-x_{1}$. For $u>x-x_{1}$, the inequality was shown in the case $x<x_{1}$. This shows (10) in the case $z=0$.

Assume now $z \geq 1$. Then for $x_{0}<x \leq x_{0}+z$, using the definition of $\xi$, 


$$
\begin{aligned}
f_{0}\left(x_{0}\right) & +x-x_{0}-C \xi^{x-z}-\zeta \sum_{k=x_{0}-x+1}^{1} p_{k}\left[f_{0}\left(x_{0}\right)+\left(x+k-x_{0}\right)\right. \\
& \left.-C \xi^{x+k-z}\right]-\zeta \sum_{-x}^{x_{0}-x} p_{k}\left[f_{0}(x+k)-C \xi^{x+k-z}\right] \\
& -\zeta \sum_{-\infty}^{-x-1} p_{k}\left[f_{0}(0)+\eta(x+k)-C \xi^{x+k-z}\right] \\
= & (1-\zeta)\left[f_{0}\left(x_{0}\right)+x-x_{0}\right]-\zeta \mathbb{E}[Y] \\
& +\zeta \sum_{-x}^{x_{0}-x} p_{k}\left[f_{0}\left(x_{0}\right)-x_{0}-\left\{f_{0}(x+k)-(x+k)\right\}\right] \\
& +\zeta \sum_{-\infty}^{-x-1} p_{k}\left[f_{0}\left(x_{0}\right)-x_{0}-\left\{f_{0}(0)+(\eta-1)(x+k)\right\}\right] \\
= & (1-\zeta)\left[f_{0}\left(x_{0}\right)+x-x_{0}\right]-\zeta \mathbb{E}[Y] \\
& +\zeta \sum_{-x}^{x_{0}-x} p_{k}\left[f_{0}\left(x_{0}\right)-x_{0}-\left\{f_{0}(x+k)-(x+k)\right\}\right]
\end{aligned}
$$

with $f_{0}(x)=f_{0}(0)+\eta x$ for $x<0$. Because $f_{0}(x)$ is the value function for the case without tax for $x \leq x_{0}$, it is concave. Further $f_{0}\left(x_{0}+1\right)-f_{0}\left(x_{0}\right)=1$. Thus $f_{0}(x+1)-f_{0}(x)$ is decreasing and the result follows as in the case $z=0$ and $x>x_{1}$.

Next consider $x_{0}+z<x \leq x_{1}+z$. Then 


$$
\begin{aligned}
& f_{0}(x-z)+z-C \xi^{x-z} \\
& -\zeta \sum_{k=x_{0}+z-x}^{1} p_{k}\left[f_{0}(x+k-z)+z-C \xi^{x+k-z}\right] \\
& \zeta \sum_{k=x_{0}-x}^{x_{0}+z-x-1} p_{k}\left[f_{0}\left(x_{0}\right)+x+k-x_{0}-C \xi^{x+k-z}\right] \\
& -\zeta \sum_{k=-x}^{x_{0}-x-1} p_{k}\left[f_{0}(x+k)-C \xi^{x+k-z}\right] \\
& -\zeta \sum_{-\infty}^{-x-1} p_{k}\left[f_{0}(0)+\eta(x+k)-C \xi^{x+k-z}\right] \\
& =(1-\zeta)\left[f_{0}(x-z)+z\right] \\
& +\zeta \sum_{k=x_{0}+z-x}^{1} p_{k}\left(f_{0}(x-z)-f_{0}(x+k-z)\right) \\
& +\zeta \sum_{k=x_{0}-x}^{x_{0}+z-x-1} p_{k}\left[f_{0}(x-z)+z-f_{0}\left(x_{0}\right)-x-k+x_{0}\right] \\
& +\zeta \sum_{k=-x}^{x_{0}-x-1} p_{k}\left[f_{0}(x-z)+z-f_{0}(x+k)\right] \\
& +\zeta \sum_{-\infty}^{-x-1} p_{k}\left[f_{0}(x-z)+z-f_{0}(0)-\eta(x+k)\right] \\
& \geq(1-\zeta)\left[f_{0}(x-z)+z\right]-\zeta \mathbb{E}[Y] \\
& -\zeta p_{1}\left(f_{0}(x+1-z)-1-f_{0}(x-z)\right) \\
& +\zeta \sum_{k=-x}^{x_{0}-x-1} p_{k}\left[f_{0}(x-z)-(x-z)-\left\{f_{0}(x+k)-(x+k)\right\}\right] \\
& +\zeta \sum_{-\infty}^{-x-1} p_{k}\left[f_{0}(x-z)-(x-z)-f_{0}(0)-(\eta-1)(x+k)\right] .
\end{aligned}
$$

As in the case $x_{0}<x \leq x_{0}+z$, the right hand side is decreasing in $x$. At $x=x_{0}+z$, the right hand side is positive, as shown in the case $x=x_{0}+z$. The assertion follows now as in the case $x_{0}<x \leq x_{0}+z$.

Let now $x>x_{1}+z$. Consider 


$$
\begin{aligned}
g(x, z) & =f\left(x_{1}, 0\right)+z+\delta\left(x-z-x_{1}\right)-C \zeta^{x_{1}} \\
& -\zeta \sum_{k=x_{1}+z-x+1}^{1} p_{k}\left[f_{0}\left(x_{1}\right)+z+\delta\left(x+k-z-x_{1}\right)-C \xi^{x_{1}}\right] \\
& -\zeta \sum_{k=x_{0}+z-x+1}^{x_{1}+z-x} p_{k}\left[f_{0}(x+k-z)+z-C \xi^{x+k-z}\right] \\
& -\zeta \sum_{k=x_{0}-x+1}^{x_{0}+z-x} p_{k}\left[f_{0}\left(x_{0}\right)+\left(x+k-x_{0}\right)-C \xi^{x+k-z}\right] \\
& -\zeta \sum_{k=-x}^{x_{0}-x} p_{k}\left[f_{0}(x+k)-C \xi^{x+k-z}\right] \\
& -\zeta \sum_{k=-\infty}^{-x-1} p_{k}\left[f_{0}(0)+\eta(x+k)-C \xi^{x+k-z}\right] .
\end{aligned}
$$

Then

$$
\begin{aligned}
g(x+1, z+1)-g(x, z)= & 1-\zeta \mathbb{P}\left[Y \geq x_{0}-x\right] \\
& -\zeta \sum_{k=-x}^{x_{0}-x-1}\left(f_{0}(x+1+k)-f_{0}(x+k)\right)-\zeta \eta \mathbb{P}[Y<-x] \\
= & \tilde{V}(x+1)-\mathbb{E}[\tilde{V}(x+1+Y)]-\{\tilde{V}(x)-\mathbb{E}[\tilde{V}(x+Y)]\} \\
\geq & 0,
\end{aligned}
$$

where $\tilde{V}(x)$ is the solution in the case without tax. The last inequality follows from the corresponding calculation in the case without tax. Note that $g(x, 0) \geq 0$ has been proved in the case $z=0$. This shows (11) also in this case.

We need to show that our additional assumption holds. Since $\xi^{x}$ is convex, and $f_{0}(x)$ has the desired property, $f(x+1,0)-f(x, 0)$ is decreasing for $x \leq x_{0}$. Suppose there is a $x_{0}<x_{2}<x_{1}$, such that $f(x+1,0)-f(x, 0)$ is decreasing for $x \leq x_{2}$ but $f\left(x_{2}+2,0\right)-f\left(x_{2}+1,0\right)>f\left(x_{2}+1,0\right)-f\left(x_{2}, 0\right)$. Then $\tilde{\delta}=f\left(x_{2}+1,0\right)-f\left(x_{2}, 0\right) \in(\delta, 1)$. By the proof above, $f\left(x_{0}, 0\right)$ is the value function of the problem with tax rate $\tilde{\delta}$. Because $\tilde{\delta}>\delta$ we have $f\left(x_{0}, 0\right)>V\left(x_{0}, 0\right)$. On the other hand, in the problem with tax rate $\delta, f\left(x_{0}, 0\right)$ is the value of the proposed strategy with barriers $x_{0}$ and $x_{1}$, respectively. Therefore, $f\left(x_{0}, 0\right) \leq V\left(x_{0}, 0\right)$. This is a contradiction. Thus our assumption must hold.

\section{References}

1. Albrecher H, Hipp C (2007) Lundberg's risk process with tax. Blätter DGVFM 28:13-28

2. Dickson DCM (2005) Insurance risk and ruin. Cambridge University Press, Cambridge

3. Dickson DCM, Waters HR (2004) Some optimal dividends problems. ASTIN Bull 34:49-74

4. de Finetti B (1957) Su un'impostazione alternativa della teoria collettiva del rischio. Trans XVth Int Congress Actuar 2:433-443 
5. Kulenko N, Schmidli H (2008) Optimal dividend strategies in a Cramér-Lundberg model with capital injections. Insur. Math. Econ. 43:270-278

6. Schmidli H (2008) Stochastic control in insurance. Springer, London

7. Schmidli H (2016) On Capital Injections and Dividends with Tax in a Classical Risk Model. Insurance: Mathematics and Economics 71:138-144

8. Schmidli H (2017) Dividends with tax and capital injection in a spectrally negative Lévy risk model. Theory Prob Math Stat 96:171-183

9. Schmidli H (2017) On capital injections and dividends with tax in a diffusion approximation. Scand Actuar J 9:751-760

10. Schmidli H (2018) Risk theory. Springer, Cham

Publisher's Note Springer Nature remains neutral with regard to jurisdictional claims in published maps and institutional affiliations. 